\title{
Epigenetische Prozesse als Drug Targets
}

Die Ätiologie neuropsychiatrischer und neurodegenerativer Erkrankungen ist oft komplex und auf variable Kombinationen von genetischen und umweltbedingten Risikofaktoren zurückzuführen. Vor allem umweltbedingte Risikofaktoren sind oftmals schwer quantitativ bestimmbar. Epigenetische Prozesse sind wichtige Regulatoren von Genom-Umwelt-Wechselwirkungen und eröffnen daher neue Ansätze für innovative und stratifizierte Therapie von Hirnerkrankungen wie z.B. Depression, posttraumatischen Belastungsstörungen oder Morbus Alzheimer. Darüber hinaus bietet die Analyse epigenetischer Markierungen die Möglichkeit, neuartige Biomarker zu entwickeln und wichtige Beiträge zur personalisierten Medizin zu leisten. In meiner Präsentation werde ich einen Überblick über das neuartige Forschungsfeld der Neuroepigenetik geben und konkrete Beispiele für die translationa- len Forschung hervorheben. Zum Beispiel haben wir entdeckt, dass Inhibitoren von Histon-Deacetylasen, welche die epigenetische Genexpression auf Systemebene kontrollieren, Kognition im Tiermodell verbessern. Sie werden nun erstmals am Menschen getestet. Ein weiterer wichtiger Bereich, den ich in meiner Präsentation ansprechen werde, ist die Möglichkeit, epigenetische Markierungen als Biomarker zu nutzen. Hier ist unsere zentrale Hypothese, dass die verschiedenen Risikofaktoren schließlich zu einer veränderten Gen-Aktivität führen. Dies beruht auf der Annahme, dass eine korrekte Genexpression ein Kernmerkmal der zellulären Homöostase ist und dass Veränderungen, die an verschiedenen Kompartimenten der Zelle auftreten (z. B. an der Synapse), letztlich mit dem Zellkern kommunizieren und so Genexpressionsänderungen verursachen.
Interessenkonflikte

Teile unserer Forschung sind von Ono Pharmaceuticals gesponsert.

Autor

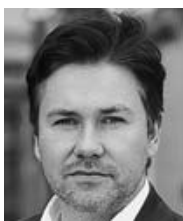

André Fischer

Deutsches Zentrum für Neurodegenerative Erkrankungen, Göttingen, Universitätsmedizin Göttingen

\section{Korrespondenzadresse}

Prof. Dr. André Fischer

Deutsches Zentrum für Neurodegenerative Erkrankungen, Göttingen

Universitätsmedizin Göttingen

Von Siebold Str. 3A

37077 Göttingen

andre.fischer@dzne.de 